\title{
Expansão, deslocamento e interiorização do homicídio no Brasil, entre 2000 e 2015: uma análise espacial
}

\author{
Expansion, displacement and interiorization of homicides in Brazil, \\ between 2000 and 2015: a spatial analysis
}

\begin{abstract}
Adauto Martins Soares Filho (https://orcid.org/0000-0002-0917-7473) ${ }^{1}$
Edgar Merchan-Hamann (https://orcid.org/0000-0001-6775-9466) ${ }^{1}$

Cintia Honório Vasconcelos (https://orcid.org/0000-0002-9635-0501) ${ }^{2}$
\end{abstract}

${ }^{1}$ Departamento de Análise da Situação de Saúde, Secretaria de Vigilância em Saúde, Ministério da Saúde. SRTVN 701, Via W5 Norte, Ed. PO700/6 DANTPS, Asa Norte. 70723-040 Brasília DF Brasil.

afilho_2006@hotmail.com

${ }^{2}$ Programa de Pós-

Graduação em Saúde

Coletiva, Universidade de

Brasília. Brasília DF Brasil.

\begin{abstract}
The scope of this paper is to analyze the variation of the spatial pattern of the homicide rate in Brazil between 2000 and 2015. It is an ecological study by micro-regions of homicides taken from the Mortality Information System, using Moran indexes, and critical area clusters (95\% CI). The rate increased by 6\% (to 29.1/100,000 inhabitants (95\% CI 28.9, 29.4), and in $80 \%$ of the micro regions between 2000 and 2015. The areas with high rates $(>38.2 / 100,000)$ increased 2.7-fold. In 2000, the highest rates were concentrated in areas in Pernambuco, São Paulo, Mato Grosso and Rio de Janeiro; by 2015, it will affect most states in the North and Northeast. The coastal regions of the Northeast and borders of Pará and Maranhão in the Amazon are critical areas. The lowest rate $(19.1 / 100,000)$ is in São Paulo and Santa Catarina micro regions, with less critical clusters in the Southeast and South regions. Homicides have expanded into the interior of Brazil, with displacement between regions, mainly gravitating towards the poorest, which exhibit more critical areas in several scenarios, such as state borders and the coast. Conversely, there is marked contraction of homicides in states of highly developed regions with the presence of less critical areas.

Key words Homicide, Violence, Conglomerate analysis, Spatial analysis
\end{abstract}

Resumo O objetivo do estudo é analisar a mudança do padrão espacial da taxa de mortalidade por homicídios (TMH) no Brasil, entre 2000 e 2015. Trata-se de estudo ecológico por microrregiões das TMH do Sistema de Informações sobre Mortalidade, utilizando os indices de Moran e clusters de áreas críticas (95\% de confiança). A TMH cresceu 6\% (para 29, 1/100 mil hab.; IC95\% 28,9; 29,4), e em $80 \%$ das microrregiões entre 2000 e 2015. O número de áreas com altas TMH (> 38,2/100 mil) aumentou 2,7 vezes. Em 2000, as áreas com TMH mais altas concentravam-se em Pernambuco, São Paulo, Mato Grosso e Rio de Janeiro; em 2015, passam a ocupar estados das regiões Norte e Nordeste. As áreas mais críticas estão no litoral do Nordeste e nas fronteiras do Pará e Maranhão na Amazônia Legal. As menores TMH (até 19,1/100 mil) estão mais presentes nas microrregiões de São Paulo e Santa Catarina, com aglomerados menos críticos nas regiões Sudeste e Sul. O homicídio se expandiu para dentro do território nacional, com deslocamento entre regiões, em direção principalmente às mais pobres, que mostram áreas mais críticas em cenários distintos, como fronteiras de estados e litoral. Inversamente, há contração expressiva do homicídio em estados de regiões de desenvolvimento alto, com presença de áreas menos críticas.

Palavras-chave Homicídio, Violência, Análise por conglomerados, Análise espacial 


\section{Introdução}

O Brasil possui 15\% (61.237) dos homicídios no mundo (taxa global de 5,5/100 mil habitantes) e está no grupo de maiores taxas de mortalidade, 27,8 por 100 mil habitantes, em 2015, dentre outras regiões da América-Latina e Caribe. Em posição oposta, países de renda ou desenvolvimento alto exibem risco reduzido ${ }^{1}$.

Nos últimos 15 anos, o Brasil ficou mais urbanizado $^{2}$ e, paralelo a esse processo, observam-se mudanças na distribuição dos homicídios entre as regiões do país. Há indícios de uma tendência recente à redução de morte violentas no Sudeste, evidentes em São Paulo e Rio de Janeiro, e crescimento nas regiões Nordeste e Norte ${ }^{3,4}$. É maior a incidência de taxa de mortalidade por homicídio (TMH) por arma de fogo, em vias públicas de áreas mais pobres de grandes cidades brasileiras, principalmente em estados da região Nordeste. Tráfico de drogas, disponibilidade de armas e desigualdades sociais são fatores contextuais que favorecem a ocorrência de homicídios ${ }^{4-6}$. Fatores de risco individuais associados a maior risco de morte são homens, jovens, negros, de baixa renda e escolaridade e consumo de álcool e drogas ${ }^{7-9}$. Mulheres assassinadas, embora em menor magnitude, apresentam perfil semelhante ${ }^{10,11}$. Interessante notar que esse padrão das vítimas não difere de forma importante do autor da violência ${ }^{12-15}$.

Ao constituir importante problema com impactos na sociedade, homicídios são considerados indicadores globais da qualidade das relações sociais e das condições de vida ${ }^{16}$. E embora os perfis das vítimas tenham sido amplamente estudados ${ }^{7-11}$, pouco se conhece sobre as tendências recentes dos homicídios no Brasil. Análise do padrão e da dinâmica de distribuição da mortalidade nas áreas geográficas do país é fundamental para o acompanhamento da mobilidade dos eventos, identificação de localidades mais vulneráveis e auxílio no levantamento de hipóteses explicativas de diferenças e fatores circunstanciais determinantes. Este estudo tem por objetivo analisar a variação do padrão espacial da mortalidade por homicídios no Brasil, entre 2000 e 2015.

\section{Métodos}

Estudo ecológico do padrão espacial de distribuição geográfica da TMH nas microrregiões do Brasil, entre 2000 e 2015. Os dados de óbitos, por local de residência, foram obtidos do Sistema de Informações sobre Mortalidade (SIM) do Minis- tério da Saúde ${ }^{17}$. As populações são do Instituto Brasileiro de Geografia e estatística (IBGE) ${ }^{18}$.

Os homicídios foram identificados pelos códigos da Classificação Internacional de Doenças (CID 10) e incluíram: agressões (X85-Y09), eventos de intenção indeterminada por arma de fogo (Y22 a Y24) e intervenções legais (Y35). As taxas foram calculadas por 100 mil habitantes, padronizadas por idade pelo método direto. A população padrão usada foi a identificada pelo censo de $2010^{18}$.

A microrregião do IBGE, que subdivide os estados brasileiros, é composta por municípios com similaridades sociais e econômicas, comumente limítrofes. E caracterizam-se como áreas que se condicionam em seus aspectos estruturais numa unidade mais orgânica de relação e interdependência. Além disso, a produção de indicadores por microrregião evita flutuações de pequenos números, observadas em municípios menores (municípios $<20$ mil hab.: $\mathrm{n}=3.823$; $69 \%)$.

A mudança ou a variação na mortalidade por homicídio por 100 mil habitantes, excluído impacto do crescimento populacional, é definida por:

Mudança $=\frac{\left(\text { óbitof }-\left(\left(\frac{\text { populaçãof }}{\text { populaçãoi }}\right) \times \text { óbitoi }\right)\right)}{\text { populaçãof }}$

Onde $f$ (final) é o dado referente ao último ano do período, e $i$ (inicial) é o dado do primeiro ano do período.

A análise espacial de dados de área foi utilizada na identificação dos padrões de dependência espacial das microrregiões para TMH, em 2000 e 2015, e para mudanças na mortalidade por homicídios no período. O índice de Moran global foi utilizado como teste para verificar a presença de cluster espacial, considerando significância de 95\%. Em seguida, aplicou-se a função de autocorrelação local (LISA - Local Indicator Spatial Association), que por sua vez indica locais onde a dependência espacial é mais pronunciada e significativamente diferente das demais. Para a análise espacial os indicadores foram normalizadas e o Moran Map foi gerado para visualização dos resultados ${ }^{19}$.

De acordo com os resultados (nível de significância à $95 \%$ de confiança), as microrregiões foram classificadas em valores de 0 a 4 , onde: 0 (não significante); 1 corresponde aos valores de Q1 (alto-alto, valores altos do indicador com vizinhança com média também alta); 2 é Q2 (bai- 
xo-baixo, valores baixos do indicador com vizinhos com média também baixa). Estas áreas são consideradas homogêneas com associação espacial positiva. Enquanto as áreas dos quadrantes 3 (Q3, alto-baixo) e 4 (Q4, baixo-alto), ou seja, valores altos do indicador próximos a vizinhos com valores baixos e vice-versa, são consideradas com associação espacial negativa, ou heterogêneas. Considerou-se área crítica, a pertencente ao quadrante Q1 (alto-alto). Antecede à análise espacial uma descrição dos indicadores georreferenciados por microrregião ${ }^{19}$.

No cálculo das TMH utilizou-se o "Programa para Análises Epidemiológicas de Dados Tabulados” (Epidata), versão 3.1, da Organização Panamericana de Saúde (OPAS)/Xunta de Galícia. Para a análise espacial foram utilizados os softwares livres TerraView 4.2.2 e QGIS 3.2.1.

$\mathrm{O}$ estudo usou exclusivamente dados secundários não nominais, em conformidade com a Resolução no $510^{20}$, de 7 de abril de 2016 que dispõe sobre as normas aplicáveis a pesquisas.

\section{Resultados}

No Brasil aumentou o homicídio entre 2000 e 2015. Ao variar de 46.698 para 59.083 óbitos, a TMH cresceu $6 \%$ para $29,1 / 100$ mil habitantes (IC95\% 28,9; 29,4). Do mesmo modo, subiu a TMH nos municípios com até 50 mil hab. (83\%; 23,2/100 mil; IC95\% 22,8; 23,5) e de 50 a 100 mil hab. (52\%; 30,1/100 mil; IC95\% 29,4; 30,8). O comportamento é inverso nos municípios com mais de 100 mil hab., que apresentaram redução de 15\% (32,1/100 mil; IC95\% 31,8; 32,4).

Entre 2000 e 2015, ocorreu aumento de microrregiões (43 para 116) pertencentes ao grupo de TMH mais elevadas (38,2 a 79,6/100 mil) no país. Em 2000, microrregiões com TMH mais elevadas se concentravam nos estados de Pernambuco (Nordeste), Mato Grosso (Amazônia Legal) e São Paulo, Rio de Janeiro e Espírito Santo, no Sudeste do país. Em 2015, as áreas com TMH elevadas aumentaram nos estados das regiões da Amazônia Legal (Norte) e Nordeste, principalmente no litoral nordestino (Figura 1a).

Em 2015, os estados com maior número de microrregiões no grupo de TMH mais elevadas foram: Sergipe (12/13), Pernambuco (11/19), Alagoas (10/13), Ceará (10/33), Bahia (10/32) e Pará (9/14). As microrregiões com maiores TMH foram: Altamira (Pará; 76,4), Catu (Bahia; 76,3), Agreste de Itabaiana (Sergipe; 75,1), Baixo Jaguaribe (Ceará; 72,7), Macaíba (Rio Grande do
Norte; 71,0) e Serrana dos Quilombos (Alagoas; 70,7). Áreas do entorno do Distrito Federal também apresentaram altas TMH, bem como amplas regiões dos estados de Goiás e Tocantins e centro-norte de Minas Gerais. Diferentemente, os estados de São Paulo (Sudeste) e Santa Catarina (Sul) apresentaram quase a totalidade de suas microrregiões nos grupos de menores $\mathrm{TMH}$ (até 19,1 por 100 mil hab.). Destaca-se que $58 \%$ das 558 microrregiões brasileiras apresentavam TMH acima de 19/100 mil hab.

De 2000 a 2015, as mudanças na mortalidade por homicídio (Figura $1 \mathrm{~b}$ ) foram crescentes para $76 \%(\mathrm{n}=422)$ das microrregiões, cujo aumento foi observado em quase toda a área de 16 dos 26 estados do país. No Nordeste, apenas Pernambuco e Piauí apresentaram áreas de redução. $\mathrm{Na}$ região Norte, cinco dos sete estados apresentaram ampla área de aumento (Pará, Amazonas, Tocantins, Amapá e Acre). E, ainda, os estados de Goiás (Centro-Oeste) e Minas Gerais (Sudeste) exibiram aumento de extensa área. Enquanto o Distrito Federal, fronteira com esses dois estados, apresentou redução. E os estados de São Paulo (53/63) e Mato Grosso do Sul (9/11) apresentaram variação decrescente para a maioria de suas microrregiões. Os demais estados estão em situação intermediária, com áreas de aumento e redução do homicídio.

O índice (I) de Moran Global evidenciou autocorrelação espacial para TMH em 2000 e 2015, e para a mudança da mortalidade por homicídio nesse período, com nível de significância de $1 \%$, e valores respectivos de $I_{2000}=0,525, I_{2015}=0,545$ e $I_{\text {mudanca }}=0,554$.

Os mapas de espalhamento de Moran exibem os Índices de Moran local das TMH (Figuras 2a) e das mudanças de mortalidade por homicidios (Figuras 2b) para cada microrregião no Brasil, visualizando aglomerados (clusters) de áreas homogêneas, estatisticamente significantes, cujos locais têm uma dependência espacial mais pronunciada.

O Moran Map das TMH (Figura 2a) identificou aglomerados críticos (Alto-Alto), formados por áreas com TMH elevadas na vizinhança de áreas com TMH também elevadas, representados por áreas mais escuras no mapa (homogêneas/associação positiva). No ano 2000 essas áreas concentravam-se nitidamente nos estados de Mato Grosso, Mato Grosso do Sul, Rondônia, Sudeste do Pará, Roraima, quase todo o estado de Pernambuco, norte de Alagoas e em toda a extensão litorânea dos estados de São Paulo e Rio de Janeiro, englobando também suas capitais, e 


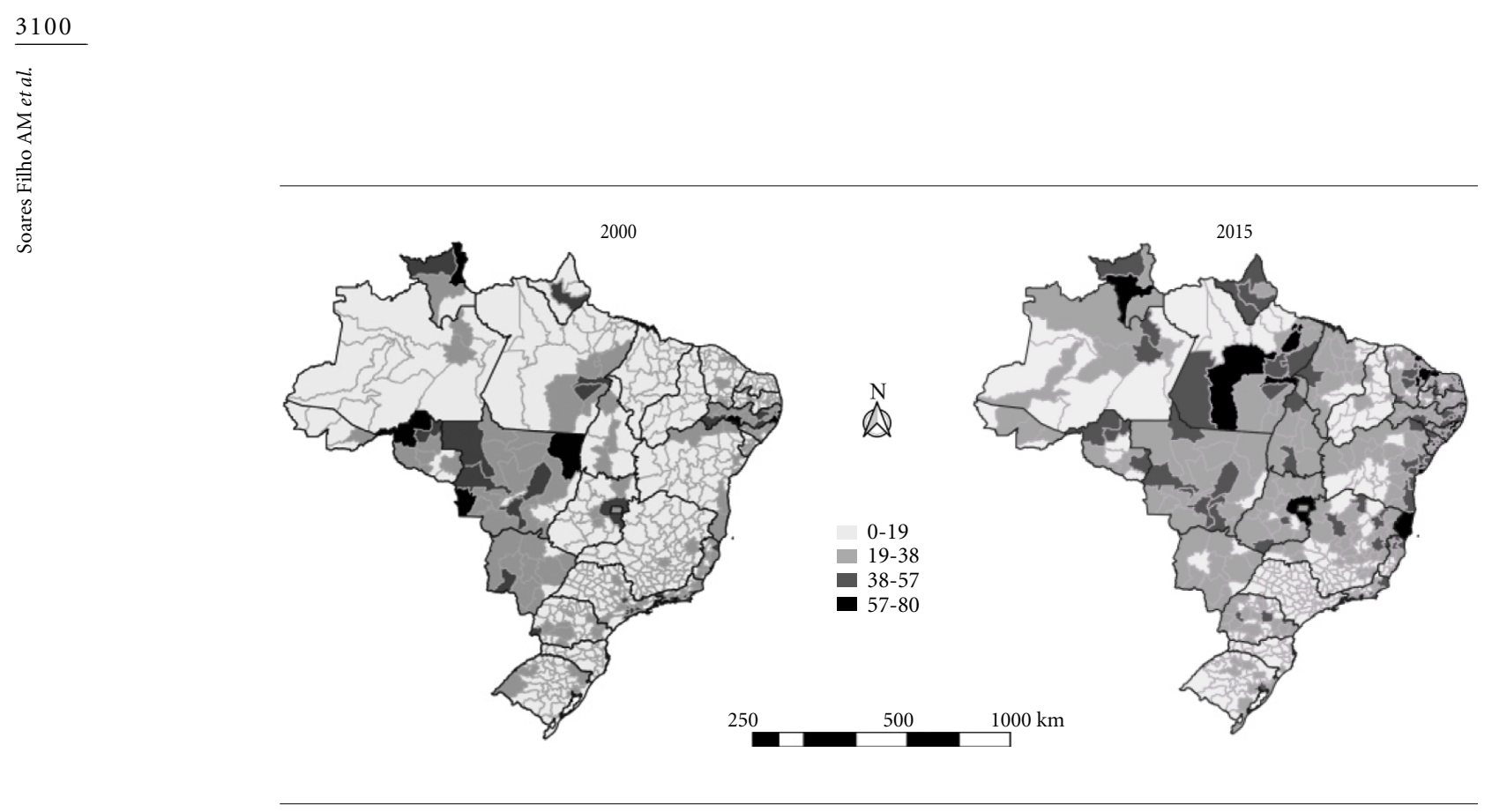

Figura 1a. Taxa ajustada de homicídios (por 100 mil habitantes) nas microrregiões, Brasil, 2000 e 2015.

Fonte: Coordenação Geral de Informações e Análises Epidemiológicas. SVS/SIM e IBGE.
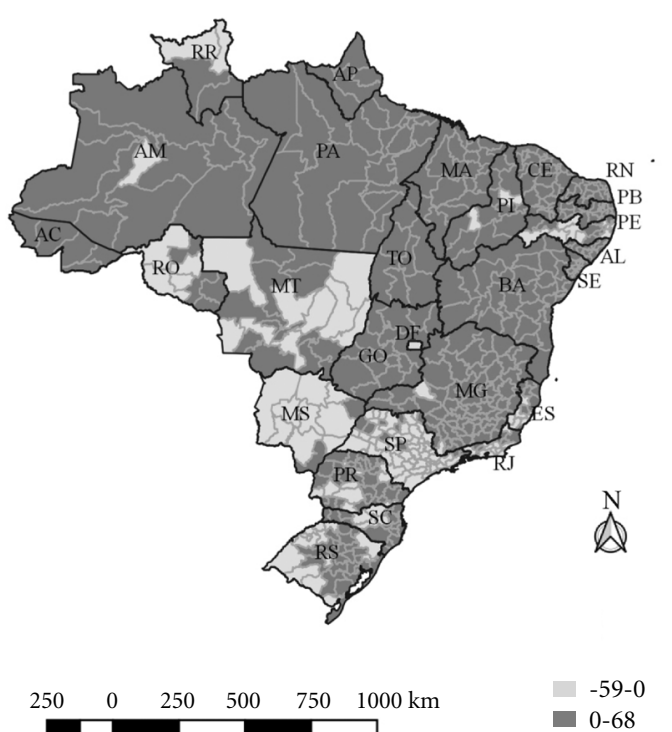

Figura 1b. Variação de homicídios (por 100 mil habitantes) nas microrregiões, Brasil, 2000 a 2015 (excluído impacto do crescimento populacional).

Fonte: Coordenação Geral de Informações e Análises Epidemiológicas. SVS/SIM e IBGE. áreas do Espírito Santo. Em 2015 essas áreas se concentravam nos estados do Nordeste, principalmente em Sergipe, Alagoas, Pernambuco (da região metropolitana ao agreste), Ceará e divisa com o Rio Grande do Norte e em todo o litoral da Bahia e norte do Espírito Santo, além do aumento no leste paraense, incluindo áreas de fronteira com Maranhão e Tocantins. Apesar da redução nos estados de Mato Grosso e Roraima, ainda permanecem áreas críticas importantes.

Outras áreas homogêneas (associação positiva de menor prioridade) são os aglomerados com baixas TMH, na vizinhança de TMH também baixas (Baixo-Baixo). Em 2000, os aglomerados de menor prioridade ocupavam boa parte dos estados do Nordeste (em particular Maranhão, Piauí e oeste da Bahia) e de Minas Gerais, além do norte do Pará, oeste do Amazonas e parte do Acre. Em 2015, o cenário mudou, concentrando áreas de menor prioridade nas regiões Sudeste e Sul; quase todo o estado de São Paulo, porção sul de Minas Gerais, e os estados de Santa Catarina e Rio Grande do Sul. Aglomerados de prioridade intermediária, cujas TMH elevadas situaram-se na vizinhança de áreas com TMH baixas e viceversa (associação negativa/heterogênea), configuraram-se áreas isoladas (Figura 2a).

Os aglomerados de áreas de mudanças da mortalidade por homicídio, entre 2000 e 2015 (Figura 2b), guardam estreita relação com as modificações de clusters das TMH no período (Fi- 


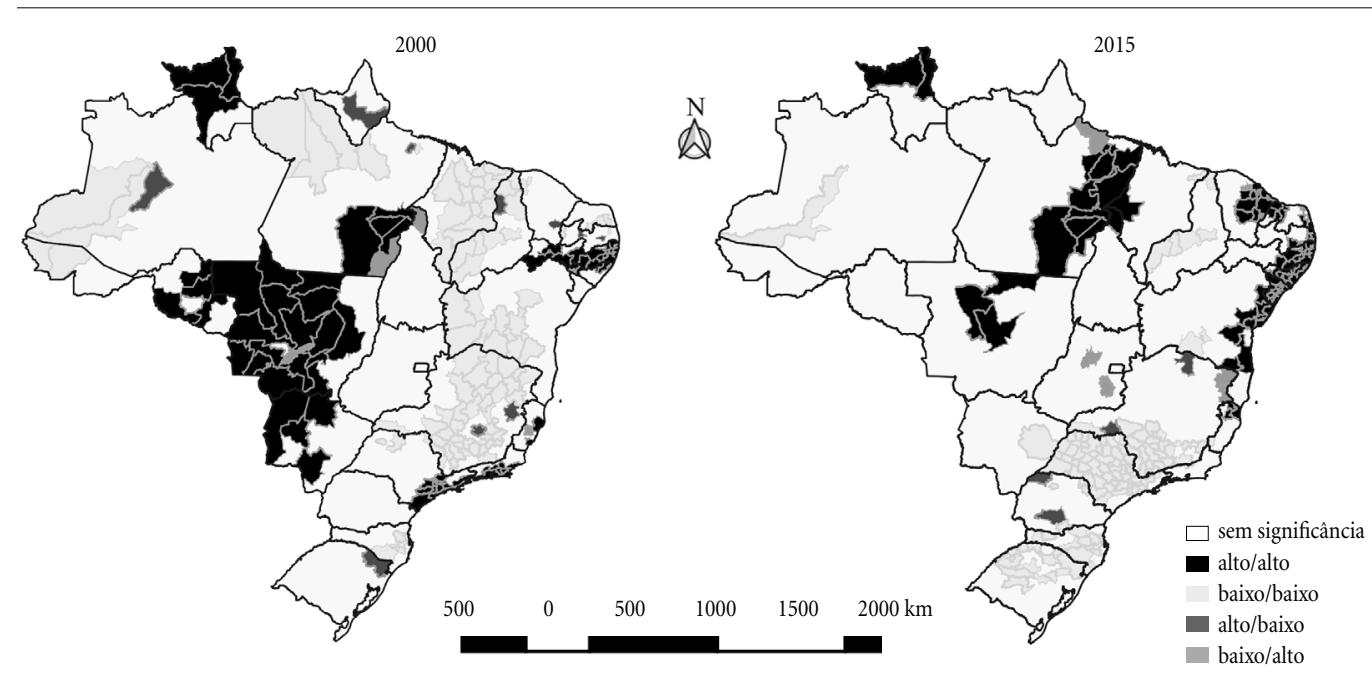

Figura 2a. Mapa de espalhamento de Moran (Moran Map) da taxa de homicídio (por 100 mil habitantes), segundo microrregião, Brasil, 2000 e 2015.

Fonte: Coordenação Geral de Informações e Análises Epidemiológicas. SVS/SIM e IBGE.

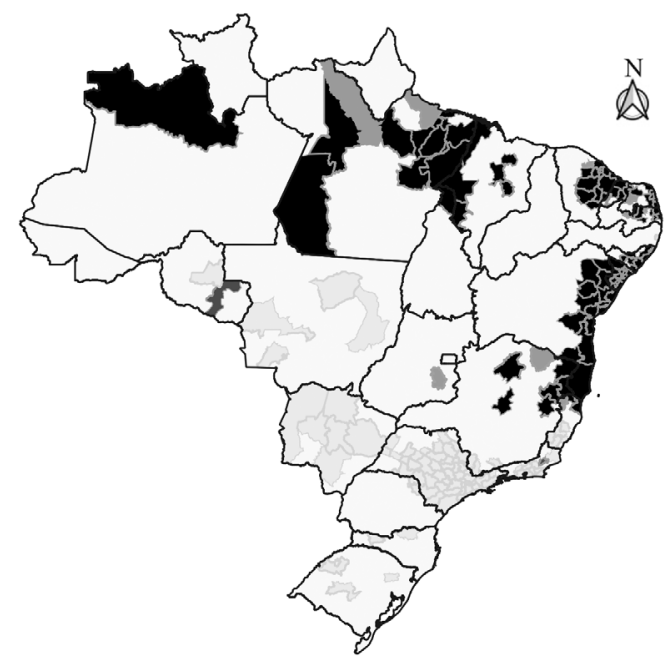

$\begin{array}{rrrrrr}250 & 0 & 250 & 500 & 750 & 1000 \\ \mathbf{k m}\end{array}$

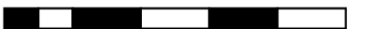

$$
\begin{aligned}
& \square \text { sem significância }(\mathrm{n}=370) \\
& \text { alto/alto ( } \mathrm{n}=92) \\
& \text { baixo/baixo }(\mathrm{n}=87) \\
& \text { alto/baixo }(\mathrm{n}=3) \\
& \text { baixo/alto }(\mathrm{n}=10)
\end{aligned}
$$

Figura 2b. Mapa de espalhamento de Moran (Moran Map) da variação de homicídios (por 100 mil habitantes), segundo microrregião, Brasil, 2000 e 2015.

Fonte: Coordenação Geral de Informações e Análises Epidemiológicas. SVS/SIM e IBGE. gura 2a). Consideradas as áreas de dependência espacial (Figura 2b), observam-se aglomerados de mudanças crescentes de homicídio (áreas críticas) em quase todos os estados do Nordeste. Nesta mesma situação, encontram-se áreas de fronteira de Minas Gerais, Bahia e Espírito Santo, o estado do Pará, com áreas de fronteira com o Maranhão e o norte do Amazonas. Aglomerados com mudanças decrescentes do homicídio (baixa prioridade) ocorrem em quase todos os estados de São Paulo e Mato Grosso do Sul e áreas específicas do Mato Grosso, Rio de Janeiro e Espírito Santo.

Entre as microrregiões classificadas como estatisticamente não significantes no Moran Map (Figuras 2a e 2b) ocorreram áreas relevantes, por apresentar áreas com altas taxas, embora sem a influência direta da vizinhança, como, por exemplo, o entorno do Distrito Federal, conforme se observa nos mapas das Figuras 1a e 1b.

\section{Discussão}

O risco de mortalidade por homicídio se expandiu com alcance ao interior do Brasil, em direção principalmente ao Nordeste e maiores faixas das regiões Centro-Oeste e Norte; com contração mais evidente em determinados estados da região Sudeste. Áreas críticas, também, deslocaram-se, e atualmente situam-se em uma faixa da 
costa brasileira, que se inicia no extremo norte do Sudeste e segue por quase toda a região Nordeste, em aglomerados contíguos da região central do país na Amazônia Legal e em área de fronteira da região Norte com a Venezuela.

O crescimento da TMH e a expansão de áreas de risco aparecem combinados ao fluxo ascendente de distribuição da população em área urbana e de sua integração com o interior do Brasil nas últimas décadas ${ }^{4,21,22}$. Nesse movimento de urbanização, destacam-se cidades médias e arredores de áreas metropolitanas, polos de atração regional, que exercem dominância socioeconômica e funcional sobre cidades menores ${ }^{23,24}$.

Fato importante é a expansão do homicídio para localidades afastadas de áreas metropolitanas, com ampliação de territórios de alto risco, principalmente no Norte e Nordeste do país. Aglomerados de áreas críticas passaram a se concentrar na faixa costeira que se prolonga da Bahia ao Ceará e aumento de áreas no Pará. Estudo realizado em meados da década de 2000 já apontava para o deslocamento, o avanço e o espalhamento de homicídios em direção a outros municípios de áreas metropolitanas e interior dos estados no Brasil ${ }^{25}$. Delitos, como assaltos, também estão em ascensão e espalham-se para localidades rurais, conforme se tornam mais integradas no espaço a áreas urbanas, enquanto serviços públicos, incluindo segurança, estão concentrados nas grandes cidades ${ }^{26}$.

Maiores TMH em estados das regiões Norte e Nordeste país coincidem com intensa e desordenada urbanização de suas capitais, marcada por crescimento econômico e desigualdades ${ }^{3,27,28}$. $\mathrm{Na}$ Bahia, semelhante a outros estudos, as TMH foram maiores no litoral do estado, nas fronteiras ao norte com Alagoas e Pernambuco e ao sul com o Espírito Santo e Minas Gerais. Territórios de fronteira como esses ajudam a explicar TMH elevadas em determinadas áreas. É possível que corredores de ligação (rodovias e municípios contíguos) entre as regiões dos estados favoreçam a interiorização da violência. Nestas áreas críticas podem ainda ter influência a centralidade e a força econômica de alguns municípios ${ }^{21,29-31}$. Outros cenários têm sofrido aumento bem seletivo de homicídios: confrontos pela posse da terra e direito de propriedade nas questões agrárias e conflitos territoriais pela cessão de terras indígenas e quilombolas ${ }^{32-35}$.

Estados da região Sul, embora com menor magnitude, apresentaram aumento nas TMH. Distingue-se, contudo, o estado do Paraná por exibir aumento de áreas com altas TMH. Concordante com achados anteriores, que incluem entre territórios críticos cidades metropolitanas na tríplice fronteira (Brasil, Paraguai, Argentina), e em trajetos que levam ao Sudeste, cujo risco de homicídio se eleva à medida que aumenta o porte do município ${ }^{36-39}$. Corredores a partir de fronteiras de entrada de drogas e armas impactam direta e indiretamente diferentes regiões, pela violência ligada ao tráfico ${ }^{40}$.

Pernambuco foi o estado que apresentou áreas de redução importante da violência no Nordeste. Bem diferente dos anos de 1980 e 1990, quando a violência crescia na área metropolitana da capital Recife e no interior, no chamado "polígono da maconha". Na capital, possivelmente a violência crescia relacionada à migração e à concentração populacional, em precárias condições socioeconômicas e de desigualdades. Já no interior, podia ser produto principalmente da chegada do plantio da maconha, condição inerente de aumento da violência ${ }^{40-42}$.

Os estados de São Paulo, Santa Catarina e Mato Grosso do Sul estão entre as unidades com menores TMH ou entre as que apresentam reduções para a maioria de suas microrregiões. Os aglomerados de menor prioridade estendem-se principalmente do sul do Espírito Santo ao Mato Grosso do Sul, passando por São Paulo e Rio de Janeiro. São Paulo e Rio de Janeiro, no entanto, foram os estados que mais contribuíram para a redução dos homicídios ${ }^{43}$.

A redução da TMH no Sudeste, eminente em São Paulo e Rio de Janeiro, pode estar relacionada a fatores que atuam associados: mudança demográfica (redução da população jovem), melhoria do nível e da distribuição de renda, aumento das oportunidades escolares e políticas de segurança (desarmamento e encarceramento) ${ }^{44-48}$. A redução do homicídio em São Paulo repercutiu em diferentes grupos, mas principalmente em homens e jovens de áreas de alta exclusão social ${ }^{3,44,45}$. O papel da segurança pública na diminuição dos homicídios parece perder importância explicativa para desemprego e proporção de jovens, o que pode responder por parte expressiva da redução ${ }^{46,47}$. Teriam ainda alguma atribuição certos dispositivos extralegais de regulação interna ao "mundo do crime" que se legitimam nas periferias das cidades ${ }^{48}$. No Rio de Janeiro, o declínio do homicídio pode ser resultado também de medidas como a implantação de polícia comunitária ${ }^{49}$.

O presente estudo foi desenvolvido com uso de dados secundários de mortalidade. Este fato encerra limitações que merecem atenção. Por exemplo, a existência de códigos inespecíficos, tais como eventos de intenção indeterminada, 
nos dados de mortalidade por causas externas, pode acarretar limitações desta análise $e^{1,17}$. No intuito de amenizar a repercussão da exclusão desses casos, optou-se, conforme descrito nos métodos, por considerar alguns desses códigos (Y22-Y24) como homicídio. Ainda assim, certo erro de classificação pode ter ocorrido. Além disso, o uso de apenas dois anos na análise das tendências das TMH pode omitir variações relevantes intraperíodos.

Observa-se certa dinâmica espacial de modificação da TMH materializada na tendência a contágio de áreas e na composição de aglomerados em localidades com algum dinamismo econômico ${ }^{43}$. Em alguma medida, essa arquitetura de reorganização da violência no território nacional oferece racionalidade explicativa acerca dos movimentos de ampliação e interiorização do homicídio sustentados nos achados deste estudo. É esperada a conformação de fatores intensificadores do homicídio e de outras violências nesses novos espaços de incidência da TMH, dado seu aspecto multicausal, marcado por: crescimento desordenado, alta proporção de jovens, precária efetivação de políticas públicas, principalmente nas periferias das cidades, num ambiente de disponibilidade de armas e tráfico, consumo de álcool e drogas, e violência domiciliar.

Acrescentam-se os territórios de fronteira, que parecem imprimir marcante função no sustento da expansão da violência. Ao colocar essas regiões como prioritárias para o desenvolvimento regional, concepções atualizadas reconhecem que o combate integrado à violência envolve as condições de vida dos cidadãos que compartilham de iguais possibilidades de superação das condições de atraso ${ }^{49}$. Essa iniciativa intergovernos sobre faixas de fronteira brasileira pode ser aplicada em alguma medida a áreas de vizinhança de estados e microrregiões dentro do país, como as que ocorrem nas divisas da Bahia com Espírito Santo e Minas Gerais, do Distrito Federal com Minas Gerais e Goiás, e do Pará com Maranhão e Tocantins.

A recente crise econômica e as perdas da capacidade financeira do Estado brasileiro, que tornam políticas sociais e de segurança cada vez mais frágeis, podem trazer importante impacto na tendência e na intensidade do risco de homicídio nos diferentes espaços. Somam-se a este macrocontexto, a crise de credibilidade oriunda da corrupção e a precária governabilidade que afetam a sustentabilidade de políticas públicas ${ }^{50}$. A compreensão da violência nesse novo contexto, sua mobilidade e seus fatores associados, nos territórios, demanda investigações que possam apontar conglomerados e eventos prioritários em cenários específicos na implementação de políticas fundamentais, que possam ser reproduzíveis e sustentáveis em variadas localidades.

\section{Colaboradores}

AM Soares Filho e CH Vasconcelos contribuíram na concepção, desenho do estudo, aquisição dos dados e, análise e interpretação dos dados. Todos contribuíram na redação das versões preliminares, aprovaram a versão final do artigo e declaram-se responsáveis por todos os aspectos do trabalho, no sentido de garantir que as questões relacionadas à exatidão ou à integridade de qualquer parte da obra sejam devidamente investigadas e resolvidas. 


\section{Referências}

1. Institute for Health Metrics and Evaluation [Internet]. Data Visualization. 2015 [acessado 2017 Ago 10]. Disponível em: http://ghdx.healthdata.org/gbd -results-tool

2. Brasil. Ministério da Saúde (MS). Indicadores e dados básicos. Indicadores demográficos: grau de urbanização. [acessado 2017 Jul 12]. Brasília: RIPSA/MS. Disponível em: http://datasus.saude.gov.br/index. php?option=com_blankcomponent\&view $=$ default\&Itemid $=631$

3. Malta DC, Minayo MCS, Soares Filho AM, Silva MMA, Montenegro MMS, Ladeira RM Morais Neto OL, Melo AP, Mooney M, Naghavi M. Mortality and years of life lost by interpersonal violence and self-harm: in Brazil and Brazilian states: analysis of the estimates of the Global Burden of Disease Study, 1990 and 2015. Rev. bras. epidemiol. 2017; 20(Supl. 1):142156.

4. Mendes LVP, Campos MR, Von-Doellinger VR, Mota JC, Pimentel TG, Schramm JMA. A evolução da carga de causas externas no Brasil: uma comparação entre os anos de 1998 e 2008. Cad Saude Publica 2015; 31(10):2169-2184.

5. Ribeiro M, Dunn J, Laranjeira R, Senso R. High mortality among young crack cocaine users in Brazil: a 5-year followup study. Adicction 2004; 99(9):11331135.

6. Tavares R, Catalan VDB, Romano MM, Melo EM. Homicídios e vulnerabilidade social. Cien Saude Colet 2016; 21(3):923-934.

7. Cruz JM. La victimización por violência urbana: niveles e factores associados en ciudades de América Latina y Espana. Rev Panam de Salud Publica 1999; 5(4/5):259-267.

8. Andreuccetti G, Carvalho HB, Carvalho Ponce J, Carvalho DG, Kahn T, Muñoz DR, Leyton V. Alcohol consumption in homicide victims in the city of São Paulo. Addiction 2009; 104(12):1998-2006.

9. Soares Filho AM. Vitimização por homicídios segundo características de raça no Brasil. Rev Saude Publica 2011; 45(4):745-755.

10. Silva LS, Meneses MLN, Lopes CLA, Correa MSM. Anos potenciais de vida perdidos por mulheres vítimas de homicídio na cidade do Recife, Pernambuco, Brasil. Cad Saude Publica 2011; 27(9):1721-1730.

11. Silva MA, Cabral Filho JE, Amorim MMR, Falbo Neto GH. Mulheres vítimas de homicídio em Recife, Pernambuco, Brasil, 2009/2010: um estudo descritivo. Cad Saude Publica 2013; 29(2):391-396.

12. Bastos MJRP, Pereira JA, Smarzaro DC, Costa EF, Bossanel RCL, Oliosa DMS, Pereira JGP, Feitosa HN, Costa MF, Oliveira FJP, Fávero JL, Maciel ELN. Análise ecológica dos acidentes e da violência letal em Vitória, ES. Rev Saude Publica 2009; 43(1):123-132.

13. Cardona M, García HI, Giraldo CA, López MV, Suárez CM, Corcho DC, Posada CH, Flórez MN. Homicidios en Medellín, Colombia, entre 1990 y 2002: actores, móviles y circunstancias. Cad Saude Publica 2005; 21(3):840-851.
14. Kodato S, Silva APS. Homicídios de adolescentes: refletindo sobre alguns fatores associados. Psicol. Reflex. Crit. 2000; 13(3):507-515.

15. Nachif MCA. Homicide as a public health problem in the city of Campo Grande, Mato Grosso do Sul, Brazil. Psicol. Soc. 2006; 18(2):99-104.

16. World Health Organization (WHO). World health statistics 2017: monitoring health for the SDGs, Sustainable Development Goals. Geneva: WHO; 2017.

17. Brasil. Ministério da Saúde (MS). Informações de saúde: estatísticas vitais. Brasília: MS. [acessado 2017 Jul 12]. Disponível em: http://tabnet.datasus.gov.br/cgi/ deftohtm.exe?sim/cnv/ext10br.def

18. Brasil. Ministério da Saúde (MS). Informações de saúde: demográficas e socioeconômicas. Brasília: RIPSA/SVS/MS. [acessado 2017 Jul 12]. Disponível em: http://tabnet.datasus.gov.br/cgi/deftohtm.exe?novapop/cnv/popbr.def

19. Bailey T, Gattrel A. Spatial Data Analysis by Example. London: Longman; 1995.

20. Brasil. Ministério da Saúde (MS). Resolução no 510, de 7 de abril de 2016. Dispõe sobre as normas aplicáveis a pesquisas em Ciências Humanas e Sociais. Diário Oficial da União 2016; 24 maio.

21. Souza TO, Pinto LW, Souza ER. Estudo espacial da mortalidade por homicídio, Bahia, 1996-2010. Rev Saude Publica 2014; 48(3):468-477.

22. Duarte EC, Garcia LP, Freitas LRS, Mansano NH, Monteiro RA, Ramalho WM. Associação ecológica entre características dos municípios e o risco de homicídios em homens adultos de 20-39 anos de idade no Brasil, 1999-2010. Cien Saude Colet 2012; 17(9):22592268.

23. Stamm C, Staduto JAR, Lima JF, Wadi YM. A população urbana e a difusão das cidades de porte médio no Brasil. Interações 2013; 14(2):251-265.

24. Gurgel APC. As metrópoles do interior do Nordeste: a caracterização de um tipo metropolitano regional. Cadernos Metrópole 2017; 19(40):841-864.

25. Souza ER, Lima MLC. The panorama of urban violence in Brazil and its capitals. Cien Saude Colet 2006; 11(2):363-373.

26. Scorzafave LG, Justus M, Shikida PFA. Safety in the global south: Criminal victimization in Brazilian rural áreas. Journal of Rural Studies 2015; 39:247-261.

27. Bando DH, Lester D. Estudo ecológico sobre suicídio e homicídio no Brasil. Cien Saude Colet 2014; 19(4):1179-1189.

28. Alves WA, Correia DS, Barbosa LLB, Lopes LM, Melânia MIASM. Violência letal em Maceió-AL: estudo descritivo sobre homicídios, 2007-2012. Epidemiol. Serv. Saúde 2014; 23(4):731-740.

29. Souza TO, Souza ER, Pinto LW. Evolução da mortalidade por homicídio no Estado da Bahia, Brasil, no período de 1996 a 2010. Cien Saude Colet 2014; 19(6):1889-1900.

30. Costa FAMM, Trindade RFC, Santos CB. Mortes por homicídios: série histórica. Rev. Latino-Am. Enfermagem 2014; 22(6):1017-1025. 
31. Andrade-Barbosa TL, Xavier-Gomes LM, Barbosa VA, Caldeira AP. Mortalidade masculina por causas externas em Minas Gerais, Brasil. Cien Saude Colet 2013; 18:711-719.

32. Bragato FF, Bigolin Neto P. Conflitos territoriais indígenas no Brasil: entre risco e prevenção. Rev. Direito Práx. 2017; 8(1):156-195.

33. Nascimento VES, Saes MSM, Zylbersztajn D. Direitos de propriedade, investimentos e conflitos de terra no Brasil: uma análise da experiência paranaense. Rev. Econ. Sociol. Rural 2010; 48(3):705-748.

34. Ayoub D. Sofrimento, tempo, testemunho: expressões da violência em um conflito de terras. Horiz. antropol. 2014; 20(42):107-131.

35. Boyer V. Ethnoterritorial reconfigurations of social conflicts: from cultural difference to political fragmentation (brazilian Amazonia). Sociol. Antropol. 2017; 7(2):395-428.

36. SM, Soares DA, Souza RKT, Matsuo T, Souza HD. Homicídios de homens de quinze a 29 anos e fatores relacionados no estado do Paraná, de 2002 a 2004. Cien Saude Colet 2011; 16(Supl. 1):1281-1288.

37. Lozada EMK. Mathias TAF, Andrade SM, Aidar T. Tendência da mortalidade por homicídios no estado do Paraná, segundo Regionais de Saúde, 1979 a 2005. Rev Bras Epidemiol. 2009; 12(2):258-269.

38. Ramão FP, Wadi YM. Espaço urbano e criminalidade violenta: análise da distribuição espacial dos homicídios no município de Cascavel/PR. Rev. Sociol. Polit. 2010; 18(35):207-230.

39. Andrade L, Nihei OK, Pelloso SM, Carvalho MDB. Homicídios juvenis e informalidade em um município brasileiro da tríplice fronteira Brasil, Paraguai e Argentina. Rev Panam Salud Publica 2012; 31(5):380387.

40. Fiore M. O lugar do Estado na questão das drogas: o paradigma proibicionista e as alternativas. Novos estud. - CEBRAP 2012; 92:9-21.

41. Lima MLC, Souza ER, Ximenes R, Albuquerque MFPM, Bitound J, Barros MDA. Evolução de homicídios por área geográfica em Pernambuco entre 1980 e 1998. Rev Saude Publica 2002; 36(4):462-469.

42. Lima MLC, Ximenes RA, Feitosa CL, Souza ER, Albuquerque MFPM, Barros MDA, Souza WV, Lapa TM. Conglomerados de violência em Pernambuco, Brasil. Rev Panam Salud Publica 2005; 18(2):122-128.

43. Andrade LT, Diniz AMA. A reorganização espacial dos homicídios no Brasil e a tese da interiorização. R. Bras. Est. Pop. 2013; 30(Supl.):S171-S191.

44. Peres MFT, Almeida JF, Vicentin D, Cerda M, Cardia N, Adorno S. Queda dos homicídios no Município de São Paulo: uma análise exploratória de possíveis condicionantes. Rev Bras Epidemiol 2011; 14(4):709-721.

45. Nadanovsky P. O aumento no encarceramento e a redução nos homicídios em São Paulo, Brasil, entre 1996 e 2005. Cad Saude Publica 2009; 25(8):18591864.
46. Peres MFT, Almeida JF, Vicentin D, Ruotti C, Nery MB, Cerda M, Cardia N, Adorno S. Evolução dos homicídios e indicadores de segurança pública no $\mathrm{Mu}$ nicípio de São Paulo entre 1996 a 2008: um estudo ecológico de séries temporais. Cien Saude Colet 2012; 17(12):3249-3257.

47. Mello JMP, Schneider A. Mudança Demográfica e a Dinâmica dos Homicídios no Estado de São Paulo. São Paulo em Perspectiva 2007; 21(1):19-30.

48. Barcellos C, Zaluar A. Homicídios e disputas territoriais nas favelas do Rio de Janeiro. Rev Saude Publica 2014; 48(1):94-102.

49. Gadelha CAG, Costa L. Integração de fronteiras: a saúde no contexto de uma política nacional de desenvolvimento. Cad Saude Publica 2007; 23(Supl.):S214-S226.

50. Barbosa Filho FH. A crise econômica de 2014/2017. Estud. Av. 2017; 31(89):51-60.

Artigo apresentado em 29/08/2018

Aprovado em 30/11/2018

Versão final apresentada em 02/12/2018 
